\title{
A Randomized Clinical Trial of Nutrition Education for Improvement of Diet Quality and Inflammation in Iranian Obese Women
}

\author{
Majid Mohammadshahi, ${ }^{1}$ Fatemeh Haidari, ${ }^{2}$ Majid Karandish, ${ }^{2}$ \\ Sara Ebrahimi, ${ }^{3}$ and Mohammad-Hosein Haghighizadeh ${ }^{4}$ \\ ${ }^{1}$ Hyperlipidemia Research Center, Ahvaz Jundishapur University of Medical Sciences, Ahvaz 61357-15794, Iran \\ ${ }^{2}$ Nutrition and Metabolic Diseases Research Center, Ahvaz Jundishapur University of Medical Sciences, Ahvaz 61357-15794, Iran \\ ${ }^{3}$ Department of Nutritional Science, Arvand International Division of Ahvaz Jundishapur University of Medical Sciences, \\ Ahvaz 61357-15794, Iran \\ ${ }^{4}$ School of Health, Ahvaz Jundishapur University of Medical Sciences, Ahvaz 61357-15794, Iran
}

Correspondence should be addressed to Sara Ebrahimi; saraebrahimi123@gmail.com

Received 25 June 2014; Accepted 20 September 2014; Published 2 October 2014

Academic Editor: H. K. Biesalski

Copyright @ 2014 Majid Mohammadshahi et al. This is an open access article distributed under the Creative Commons Attribution License, which permits unrestricted use, distribution, and reproduction in any medium, provided the original work is properly cited.

Background. Obesity is considered as a low grade inflammation condition. The aim of this study was to investigate the effect of nutritional education on diet quality and biomarkers of inflammation in Iranian obese women. Method. Sixty obese women voluntarily participated in this randomized clinical trial and were randomly assigned to intervention or control group $(n=30)$. Intervention group was instructed to attend nutrition education sessions ( $1 \mathrm{hr} / \mathrm{wk}$, for 3 months) in small groups. Diet quality scores were measured by Healthy Eating Index (HEI). Anthropometric indices and serum concentration of hs-CRP, TNF- $\alpha$, and adiponectin were measured at the baseline and end of the intervention. Results. There were no significant differences in anthropometric indices of participants between the two groups at the end of intervention $(P>0.05)$. However, the total HEI score was significantly higher in the educated group compared to the control group after intervention $(P<0.05)$. The educated group also showed significant lower concentration of TNF- $\alpha$ and hs-CRP and higher levels of adiponectin than the control group at the end of study $(P<0.05)$. Conclusions. Our results provide limited evidence that higher dietary quality contributes to reduced inflammation in obese women. This effect could be independent of the weight loss.

\section{Background}

The link between unhealthy eating habits and obesity is well established [1-4]. Since human diets contain many components that may work synergistically to prevent or promote disease, assessing that diet quality may be an informative strategy when studying the relation between nutrition and obesity [5-8].

Indexes of dietary quality have been developed to address this need in nutrition research. Two of these, the Healthy Eating Index (HEI) and the Diet Quality Index (DQI), were developed to measure adherence to dietary guidelines and were shown to adequately measure overall diet quality $[9,10]$. The HEI measures adherence to the Food Guide Pyramid developed in the mid-1990s, whereas the DQI reflects a person's adherence to the Diet and Health recommendations of the National Academy of Sciences [11].

Obesity is a low-grade inflammatory condition; however, limited studies to date have considered the overall quality of the diet and its relation to obesity-induced inflammation; these have been mainly limited to the cross-sectional studies $[1,12,13]$. According to findings from the National Health and Nutrition Examination Survey III, the HEI score was inversely associated with C-reactive protein (CRP) concentration, which was largely attributed to total grain consumption [13]. Fung et al., however, found no association between HEI score and various markers of inflammation and endothelial dysfunction [14]. Fargnoli et al. showed 
that adherence to a healthier diet, as reflected by a higher HEI score, is associated with higher plasma adiponectin and lower plasma resistin, CRP, and E-selectin levels independent of obesity and lifestyle factors [2]. Thus, dietary quality may be inversely associated with inflammatory conditions independent of BMI [12].

Although concentrations of inflammatory markers and adipokines were previously associated with modifiable lifestyle factors (or dietary patterns) in cross-sectional studies, diet quality has not been previously studied in relation to proinflammatory factors and adiponectin during nutritional intervention. According to Tehran Lipid and Glucose Study (TLGS), the diet quality of most people in Tehran, Iran, (74\%) needs improvement and thus nutritional education to improve diet quality is recommended [15].

The purpose of the study reported here was to evaluate if nutrition education could improve diet quality, as measured by HEI score, and thereby it would be independent of weight loss associated with improving of inflammatory markers (hsCRP and TNF- $\alpha$ ) and adiponectin concentration.

\section{Methods}

2.1. Study Setting and Design. This study was designed as a randomized clinical trial to investigate the effects of nutritional education for 3 months on the improvement of HEI scores and serum levels of inflammatory markers in obese women. At first, a call for participating in the study was provided and the aim of the study was described completely to who attended to the Health and Nutrition Clinic of Ahvaz, Iran. Then regarding the inclusion and exclusion criteria, subjects were selected. Inclusion criteria were women with body mass index $(\mathrm{BMI}) \geq 30$, having an initial HEI score $<80$, under conditions of weight stability for at least 3 months, and subject satisfaction. Exclusion criteria included chronic disease (diabetes, cardiovascular disease, cancer, arthritis, lung disease, asthma, or serious allergies), being active in exercise or weight reduction programs, taking weight loss medications, taking drugs that are known to affect the immune system, including nonsteroidal anti-inflammatory medications and corticosteroids, consumption of nutritional supplements at least 6 months before sampling, alcohol consumption, vegan diet, smoking, and lack of subject followup (being absent in 2 or more education sessions). We also excluded under and over reporters of energy intakes [15].

\subsection{Study Population, Sample Size, and Sampling. Finally,} sixty obese women, aged $20-45 \mathrm{y}$, who had volunteered, were randomly divided into two groups (education group: $n=$ 30 and noneducation group: $n=30$ ) using a table of random numbers with block size not revealed to the investigators. Those randomized to the intervention (education) group were instructed to attend nutrition education sessions ( $1 \mathrm{hr} /$ wk for 3 months, 12 sessions) in small groups (6 persons per session) conducted by a registered dietitian. All subjects in this group received the same information over the course of 3 months of participation. Each $1 \mathrm{hr}$ session included an oral presentation on nutrition information relevant to healthy diet and HEI recommendations. In this study, the nutrition education program was based on the modified My Plate (regarding to Iranian food culture) and food pyramid guideline. All participants in the education group were also provided with a designated booklet related to nutrition education. The control group in this study did not receive the nutrition education program and they were educated for food recording only.

2.3. Data Collection and Procedure. In this study, all participants were requested not to change their physical activity habits during the study. At baseline and the end of the study, anthropometric measurements (weight, height, waist circumference (WC), and hip circumference (HC)) were measured and then waist-to-hip ratio (WHR) was determined. Body mass index (BMI) was calculated from measured height and weight. Participants' body fat percent was also measured by body state set (Quad Scan 4000).

Dietary intakes were assessed by the seven consecutive days' food record. On the basis of 7 days' food record, each HEI component score was calculated for each individual. The HEI total score was also calculated as the sum of these scores over the 12 components. Then, for each component score and for the total score, the mean score was taken. Briefly, the HEI-2005 comprises 12 components, which are scored on a scale from 0 to maximum $(M)$, where $M$ is 5,10 , or 20 according to the component. Thus, the composite HEI score can potentially range from a minimum of zero to a maximum score of 100, with 100 points referring to perfect diet quality and lower results indicating larger deviations from the recommended intakes [7]. For each individual and each component, the ratio of the reported intake of food group (relevant to the HEI component considered) to the reported energy intake was also calculated. Then, the mean of these ratios over the individuals was taken. All of the dietary and anthropometric data were collected by an expert registered dietitian. In this study, a modified Nutritionist IV program was used to estimate dietary intake of participants.

2.4. Biochemical Analysis. At baseline and the end of the study, fasting blood samples were also collected from all the participants, and sera were separated for biochemical analyses. Serum concentrations of hs-CRP, TNF- $\alpha$, and adiponectin were measured using a commercially available enzyme-linked immunosorbent assays (ELISA) method (Labor Diagnostika Nord for hs-CRP and Orgenium laboratories-Finland for TNF- $\alpha$ and adiponectin). All assays were performed according to the manufacturer's instructions.

2.5. Statistical Analysis. All statistical analyses were performed with Statistical Package for Social Sciences (SPSS Inc., Chicago, IL, USA) Program version 18 for windows. At first, normal distribution of all variables was checked with the Kolmogorov-Smirnov test. Group variable means were compared with each other using both independent sample $t$-test and ANCOVA in the adjusted models, which were adjusted for confounders (age, weight, and energy intake). The end values of each variable were also compared with the 
TABLE 1: Sociodemographic and anthropometric characteristics of the study groups at the baseline and end of study.

\begin{tabular}{|c|c|c|c|c|}
\hline Variables & Educated group $(n=30)$ & Noneducated group $(n=30)$ & $P 1$ & $P 2$ \\
\hline Age (y) & $33.13 \pm 5.60$ & $35.13 \pm 4.97$ & 0.211 & - \\
\hline Income $[n(\%)]$ & & & 0.516 & 0.306 \\
\hline Low & $4(13.33)$ & $6(19.98)$ & & \\
\hline Medium & $22(73.26)$ & $19(63.27)$ & & \\
\hline High & $4(13.33)$ & $5(16.65)$ & & \\
\hline Educational level $[n(\%)]$ & & & 0.911 & 0.783 \\
\hline$<6$ years & $1(3.33)$ & 0 & & \\
\hline $6-12$ years & $5(16.65)$ & $7(23.31)$ & & \\
\hline$>12$ years & $24(79.92)$ & $23(76.59)$ & & \\
\hline Physical activity $[n(\%)]$ & & & 0.852 & 0.771 \\
\hline Light & $22(73.26)$ & $21(69.93)$ & & \\
\hline Moderate & $6(19.98)$ & $8(26.64)$ & & \\
\hline Heavy & $2(6.66)$ & $1(3.33)$ & & \\
\hline \multicolumn{5}{|l|}{ Weight (kg) } \\
\hline Baseline & $91.1 \pm 10.6$ & $92.5 \pm 14.6$ & 0.672 & 0.581 \\
\hline End & $89.8 \pm 12.8$ & $91.8 \pm 13.5$ & 0.533 & 0.671 \\
\hline$P 3$ & 0.145 & 0.348 & & \\
\hline \multicolumn{5}{|l|}{ BMI } \\
\hline Baseline & $34.9 \pm 3.9$ & $34.7 \pm 5.07$ & 0.877 & 0.676 \\
\hline End & $34.3 \pm 4.3$ & $34.5 \pm 5.11$ & 0.852 & 0.845 \\
\hline P3 & 0.090 & 0.466 & & \\
\hline \multicolumn{5}{|l|}{$\mathrm{WC}(\mathrm{cm})$} \\
\hline Baseline & $107.3 \pm 10.8$ & $107.6 \pm 13.2$ & 0.910 & 0.721 \\
\hline End & $106.2 \pm 8.3$ & $108.2 \pm 13.5$ & 0.510 & 0.511 \\
\hline$P 3$ & 0.161 & 0.416 & & \\
\hline \multicolumn{5}{|l|}{$\mathrm{HC}(\mathrm{cm})$} \\
\hline Baseline & $110.2 \pm 10.4$ & $108.8 \pm 10.1$ & 0.593 & 0.804 \\
\hline End & $109.5 \pm 7.6$ & $109 \pm 9.9$ & 0.812 & 0.973 \\
\hline$P 3$ & 0.744 & 0.832 & & \\
\hline \multicolumn{5}{|l|}{ WHR } \\
\hline Baseline & $0.97 \pm 0.10$ & $0.98 \pm 0.06$ & 0.655 & 0.690 \\
\hline End & $0.97 \pm 0.09$ & $0.99 \pm 0.07$ & 0.417 & 0.553 \\
\hline$P 3$ & 0.802 & 0.424 & & \\
\hline \multicolumn{5}{|l|}{ Fat percent $(\%)$} \\
\hline Baseline & $34.6 \pm 6.3$ & $33.5 \pm 7.2$ & 0.211 & 0.189 \\
\hline End & $33.8 \pm 6.6$ & $33.4 \pm 6.2$ & 0.425 & 0.385 \\
\hline$P 3$ & 0.253 & 0.837 & & \\
\hline
\end{tabular}

BMI: body mass index; WC: waist circumference; HC: hip circumference; and WHR: waist to hip ratio.

Data were expressed as mean \pm SD or percentages.

$P 1$ resulted from independent sample $t$-test or chi-squared test as appropriate; $P 2$ resulted from ANCONA test (adjustment for age and energy intake); and $P 3$ resulted from paired sample $t$-test.

baseline values using paired sample $t$-test. The differences with $P$ values $<0.05$ were considered as significant.

2.6. Ethnic. The study was approved by and performed under the guidelines of the Research Ethics Committee of Ahvaz Jundishapur University of Medical Sciences, Iran (ETH-9115). A written consent was also obtained from all the participants.

\section{Results}

In this study, 60 participants with $34.15 \pm 5.34$ years old were included in the study. Sociodemographic and anthropometrics characteristics of the study participants at baseline and the end of the study are described in Table 1 . There were no significant differences in baseline and end of study measures 
TABLE 2: Dietary intakes of the study groups at the baseline and end of study.

\begin{tabular}{|c|c|c|c|c|}
\hline Variables & Educated group $(n=30)$ & Noneducated group $(n=30)$ & $P 1$ & $P 2$ \\
\hline \multicolumn{5}{|c|}{ Energy (Kcal) } \\
\hline Baseline & $2232 \pm 593$ & $2086 \pm 320$ & 0.086 & 0.105 \\
\hline End & $2041 \pm 315$ & $1931 \pm 249$ & 0.140 & 0.246 \\
\hline$P 3$ & 0.066 & 0.073 & & \\
\hline \multicolumn{5}{|l|}{ Protein (gr) } \\
\hline Baseline & $86.8 \pm 25.8$ & $84.1 \pm 16.4$ & 0.632 & 0.483 \\
\hline End & $81.4 \pm 12.5$ & $79.4 \pm 14.2$ & 0.620 & 0.909 \\
\hline$P 3$ & 0.234 & 0.061 & & \\
\hline \multicolumn{5}{|l|}{ Protein $(\%)$} \\
\hline Baseline & $14.8 \pm 2.3$ & $16.0 \pm 1.7$ & 0.091 & 0.063 \\
\hline End & $16.0 \pm 1.4$ & $16.5 \pm 2.1$ & 0.307 & 0.242 \\
\hline$P 3$ & 0.324 & 0.651 & & \\
\hline \multicolumn{5}{|c|}{ Carbohydrate (gr) } \\
\hline Baseline & $260.2 \pm 78.5$ & $219 \pm 44.2$ & 0.116 & 0.522 \\
\hline End & $206.7 \pm 40.7$ & $196 \pm 37.1$ & 0.297 & 0.103 \\
\hline$P 3$ & 0.001 & 0.006 & & \\
\hline \multicolumn{5}{|c|}{ Carbohydrate (\%) } \\
\hline Baseline & $44.5 \pm 6.3$ & $42.0 \pm 5.5$ & 0.071 & 0.132 \\
\hline End & $40.4 \pm 4.0$ & $40.6 \pm 5.8$ & 0.202 & 0.731 \\
\hline$P 3$ & 0.001 & 0.082 & & \\
\hline \multicolumn{5}{|l|}{ Fat (gr) } \\
\hline Baseline & $109.4 \pm 30.2$ & $101.7 \pm 18.4$ & 0.240 & 0.407 \\
\hline End & $73.3 \pm 18.7$ & $97 \pm 15.7$ & 0.000 & 0.000 \\
\hline$P 3$ & 0.000 & 0.045 & & \\
\hline \multicolumn{5}{|l|}{ Fat (\%) } \\
\hline Baseline & $34.6 \pm 6.3$ & $33.5 \pm 7.2$ & 0.201 & 0.083 \\
\hline End & $33.8 \pm 6.6$ & $33.4 \pm 6.2$ & 0.251 & 0.206 \\
\hline$P 3$ & 0.132 & 0.282 & & \\
\hline
\end{tabular}

Data were expressed as mean $\pm \mathrm{SD}$.

$P 1$ resulted from independent sample $t$-test; $P 2$ resulted from ANCONA test in the adjusted models; and $P 3$ resulted from paired sample $t$-test.

of participants (age, income, education, physical activity, weight, BMI, WC, HC, WHR, and fat percent) between the two groups. The anthropometric indices did not also decrease within groups at the end of study when compared to the baseline values.

Dietary intakes of the study participants are presented in Table 2. Total carbohydrate and total fat intake of participants decreased at the end of the study compared to the educated and the noneducated groups at baseline $(P<0.05)$. At the end of study, dietary intakes of total fat also decreased significantly in the educated group when compared to the control group $(P=0.000)$. This result remained significant after adjustment for confounding variables (age and energy intake).

As shown in Table 3, the mean total HEI score was $60.58 \pm$ 6.31 in educated group and $62.05 \pm 5.7$ in noneducated group at the baseline. Therefore, the group differences in baseline total HEI scores were not significant in this respect. Mean component scores were not significantly different between the two groups at the baseline. At the end of study, the total fruits, total vegetables, dark green and orange vegetables and legumes, whole grains, milk, meat and beans, saturated fats, sodium, and SoFAAS (calories from solid fats, alcoholic beverages, and added Sugars) component scores and consequently the total HEI score were significantly higher in the educated group compared to the control $(P<$ 0.05). These results remained significant after adjustment for confounders. After 3 months of nutrition education, the mean total HEI score was also significantly increased in the educated group, compared to baseline values $(83.34 \pm 5.12$ versus $60.58 \pm 6.31, P=0.000)$.

The relative intake of each HEI component to the energy intake is presented in Table 4. The average intake of total fruits, whole fruits, total vegetables, dark green and orange vegetables and legumes, total grains, whole grains, and milk per $1,000 \mathrm{kcal}$ significantly increased after intervention in the educated group compared to baseline intakes $(P<0.05)$. Furthermore, the relative intake of oils, saturated fat, and sodium components significantly decreased in this group $(P<0.05)$. After intervention, the comparison between two groups also showed that the relative intake of total fruits, whole fruits, total vegetables, dark green and orange 
TABLE 3: Component score of HEI in the study groups at the baseline and end of study.

\begin{tabular}{|c|c|c|c|c|}
\hline Components of HEI & Educated group $(n=30)$ & Noneducated group $(n=30)$ & $P 1$ & $P 2$ \\
\hline \multicolumn{5}{|l|}{ Total fruit } \\
\hline Baseline & $3.10 \pm 1.4$ & $3.14 \pm 1.09$ & 0.898 & 0.322 \\
\hline End & $4.91 \pm 0.33$ & $3.78 \pm 1.17$ & 0.000 & 0.000 \\
\hline P3 & 0.000 & 0.110 & & \\
\hline \multicolumn{5}{|l|}{ Whole fruit } \\
\hline Baseline & $4.27 \pm 0.85$ & $4.34 \pm 1.25$ & 0.797 & 0.872 \\
\hline End & $4.77 \pm 0.69$ & $4.77 \pm 0.69$ & 0.734 & 0.500 \\
\hline$P 3$ & 0.003 & 0.057 & & \\
\hline \multicolumn{5}{|l|}{ Total vegetables } \\
\hline Baseline & $3.18 \pm 1.14$ & $3.44 \pm 1.02$ & 0.354 & 0.119 \\
\hline End & $4.68 \pm 0.52$ & $3.28 \pm 0.98$ & 0.000 & 0.000 \\
\hline$P 3$ & 0.000 & 0.400 & & \\
\hline \multicolumn{5}{|c|}{ Dark green and orange vegetables and legumes } \\
\hline Baseline & $1.53 \pm 1.41$ & $1.64 \pm 1.27$ & 0.750 & 0.960 \\
\hline End & $4.36 \pm 0.86$ & $1.64 \pm 1.13$ & 0.000 & 0.000 \\
\hline$P 3$ & 0.000 & 0.598 & & \\
\hline \multicolumn{5}{|l|}{ Total grain } \\
\hline Baseline & $4.92 \pm 0.22$ & $4.80 \pm 0.35$ & 0.802 & 0.792 \\
\hline End & $4.94 \pm 0.47$ & $4.93 \pm 0.33$ & 0.858 & 0.702 \\
\hline$P 3$ & 0.873 & 0.532 & & \\
\hline \multicolumn{5}{|l|}{ Whole grain } \\
\hline Baseline & $0.4 \pm 0.1$ & $0.13 \pm 0.07$ & 0.036 & 0.021 \\
\hline End & $3.1 \pm 1.3$ & $0.13 \pm 0.49$ & 0.000 & 0.000 \\
\hline$P 3$ & 0.000 & 0.256 & & \\
\hline \multicolumn{5}{|l|}{ Milk } \\
\hline Baseline & $0.89 \pm 0.62$ & $0.77 \pm 0.34$ & 0.344 & 0.099 \\
\hline End & $5.52 \pm 2.23$ & $0.64 \pm 0.53$ & 0.000 & 0.000 \\
\hline$P 3$ & 0.000 & 0.242 & & \\
\hline \multicolumn{5}{|l|}{ Meat and beans } \\
\hline Baseline & $6.44 \pm 1.95$ & $6.36 \pm 2.31$ & 0.881 & 0.329 \\
\hline End & $7.86 \pm 2.03$ & $5.9 \pm 1.99$ & 0.000 & 0.000 \\
\hline$P 3$ & 0.005 & 0.237 & & \\
\hline \multicolumn{5}{|l|}{ Oils } \\
\hline Baseline & $7.25 \pm 1.81$ & $7.60 \pm 1.12$ & 0.377 & 0.429 \\
\hline End & $8.41 \pm 2.25$ & $8.32 \pm 1.15$ & 0.843 & 0.754 \\
\hline$P 3$ & 0.025 & 0.032 & & \\
\hline \multicolumn{5}{|l|}{ Saturated fat } \\
\hline Baseline & $7.38 \pm 1.54$ & $7.31 \pm 1.66$ & 0.849 & 0.544 \\
\hline End & $8.65 \pm 1$ & $7.56 \pm 1.41$ & 0.001 & 0.000 \\
\hline$P 3$ & 0.001 & 0.520 & & \\
\hline \multicolumn{5}{|l|}{ Sodium } \\
\hline Baseline & $4.12 \pm 2.76$ & $5.80 \pm 3.44$ & 0.040 & 0.028 \\
\hline End & $6.97 \pm 2.54$ & $4.61 \pm 3.15$ & 0.002 & 0.024 \\
\hline$P 3$ & 0.000 & 0.031 & & \\
\hline \multicolumn{5}{|l|}{ SoFAAS } \\
\hline Baseline & $17.20 \pm 2.12$ & $16.66 \pm 1.97$ & 0.211 & 0.487 \\
\hline End & $19.10 \pm 1.25$ & $17.76 \pm 1.83$ & 0.002 & 0.002 \\
\hline$P 3$ & 0.000 & 0.051 & & \\
\hline
\end{tabular}


TABLe 3: Continued.

\begin{tabular}{lccr}
\hline Components of HEI & Educated group $(n=30)$ & Noneducated group $(n=30)$ & $P 1$ \\
\hline Total score & & & \\
Baseline & $60.58 \pm 6.31$ & $62.05 \pm 5.7$ & 0.350 \\
End & $83.34 \pm 5.12$ & $63.44 \pm 7.49$ & 0.283 \\
P3 & $\mathbf{0 . 0 0 0}$ & 0.203 & $\mathbf{0 . 0 0 0}$ \\
\hline
\end{tabular}

SoFAAS.

Calories from solid fats, alcoholic beverages, and added sugars.

Data were expressed as mean $\pm \mathrm{SD}$.

$P 1$ resulted from independent sample $t$-test; $P 2$ resulted from ANCONA test (adjustment for age and energy intake); and $P 3$ resulted from paired sample $t$ test.

vegetables and legumes, total grain, whole grains, milk, and meet and beans per $1,000 \mathrm{kcal}$ was significantly higher in the educated group. In contrast, a significantly lower relative intake of oils, saturated fat, sodium, and SoFAAS per $1,000 \mathrm{kcal}$ was observed in the educated group, compared to the control group, at the end of study $(P<0.05)$. These differences even remained significant after adjustment for confounders.

As presented in Table 5, significant differences were observed between the two groups regarding serum levels of inflammatory biomarkers at the end of intervention. Serum levels of TNF- $\alpha$ and hs-CRP were significantly lower in the educated group compared to the control group $(P=$ 0.044 and $P=0.021$, resp.). This significance remained even after adjustment for age, weight, and energy intake ( $P=0.011$ and $P=0.039$, resp.). The educated group also showed significant higher adiponectin concentrations compared to the control group at the end of study $(P=$ 0.035 , in the adjusted models). Statistical analyses (within groups) also showed that the serum levels of TNF- $\alpha$ and hsCRP significantly decreased and the mean concentration of adiponectin significantly increased after intervention in the educated group $(P<0.05)$.

\section{Discussion}

The results of this study showed that 3-month nutrition education improved diet quality, as measured by the HEI. Consequently, the improved diet quality was associated with the lower levels of hs-CRP and TNF- $\alpha$ and the higher levels of adiponectin in obese women $(P<0.05)$. This effect was independent of weight changes.

The relationship of diet quality to obesity has been inconsistent, and lack of association between diet quality and anthropometric indicators of obesity has also been reported in other studies $[1,7,16-19]$. In a cross-sectional study conducted by Boynton et al., the HEI scores were modestly correlated with BMI, yet not with percent body fat in postmenopausal women. However, individuals with lower BMI or lower percent body fat had more healthful diets, as measured by the DQI [1]. One possible reason for these discrepant results is that while the DQI generally emphasizes moderation, half of the HEI score concerns meeting or exceeding the recommended amount of grains, fruits, vegetables, meat, and milk. Thus, an individual who eats more in general receives a higher HEI score, which may explain why no clear associations between HEI and body composition were observed $[1,17,18]$.

Although recent studies have shown an important role for inflammation in obesity, limited studies have evaluated the association between diet quality and circulating level of inflammatory markers with respect to obesity [20]. Since human diets contain many components that may work synergistically to prevent or promote disease, assessing diet quality may be informative [6]. The results from a crossover study that was conducted in healthy women have shown that, after adjustment for age and energy intake, women with the highest adherence to the HEI had 24\% higher plasma adiponectin and $41 \%$ lower plasma CRP than did women with the lowest adherence to the HEI. Inverse association between the HEI and TNF- $\alpha$ was also reported, but it was not significant after adjustment for body mass index [2].

Some studies have examined the contribution of major dietary patterns to markers of systemic inflammation. LopezGarcia et al., in a crossover study, showed that the prudent pattern that is characterized by higher intakes of fruit, vegetables, legumes, fish, poultry, and whole grains is inversely associated with plasma concentrations of CRP. Contrary to this finding, the Western pattern that is characterized by higher intakes of red and processed meats, sweets, desserts, French fries, and refined grains showed a positive relation with CRP and interleukin 6 after adjustment for confounders [21]. The prudent pattern and the Western pattern in Lopez-Garcia et al. study were relatively comparable to perfect diet quality and imperfect diet quality in the present study, respectively. In another study, median plasma adiponectin concentrations were $23 \%$ higher in women who most closely followed a Mediterranean-type diet than in low adherers [22]. Similar results were also reported by others [14, 23]. Similarly, Esposito et al., in a 2-y randomized controlled trial, reported that a Mediterranean-type diet accompanied by increased physical activity significantly increased adiponectin concentrations in obese postmenopausal women, even after accounting for the decreased body weight associated with the intervention [24]. The results of this study are consistent with those of the above studies and extend them by showing that adherence to a healthier diet, which is achieved by nutrition education in the educated group, was associated with higher adiponectin concentrations and lower hs-CRP and TNF- $\alpha$ levels. Several recent studies have shown that fiber, antioxidant, flavonoids, 
TABLE 4: Component relative intake of HEI in the study groups at the baseline and end of study.

\begin{tabular}{|c|c|c|c|c|}
\hline Components of HEI & Educated group $(n=30)$ & Noneducated group $(n=30)$ & $P 1$ & $P 2$ \\
\hline \multicolumn{5}{|c|}{ Total fruit (cup per $1000 \mathrm{kcal}$ ) } \\
\hline Baseline & $0.53 \pm 0.300$ & $0.51 \pm 0.19$ & 0.246 & 0.689 \\
\hline End & $1.53 \pm 0.62$ & $0.64 \pm 0.24$ & 0.000 & 0.000 \\
\hline P3 & 0.000 & 0.084 & & \\
\hline \multicolumn{5}{|c|}{ Whole fruit (cup per $1000 \mathrm{kcal}$ ) } \\
\hline Baseline & $0.25 \pm 0.12$ & $0.18 \pm 0.09$ & 0.235 & 0.315 \\
\hline End & $0.43 \pm 0.27$ & $0.20 \pm 0.29$ & 0.000 & 0.000 \\
\hline$P 3$ & 0.012 & 0.205 & & \\
\hline \multicolumn{5}{|c|}{ Total vegetables (cup per $1000 \mathrm{kcal}$ ) } \\
\hline Baseline & $0.73 \pm 0.33$ & $0.77 \pm 0.25$ & 0.565 & 0.339 \\
\hline End & $1.31 \pm 0.32$ & $0.76 \pm 0.36$ & 0.000 & 0.000 \\
\hline$P 3$ & 0.000 & 0.775 & & \\
\hline \multicolumn{5}{|c|}{ Dark green and orange vegetables and legumes (cup per $1000 \mathrm{kcal}$ ) } \\
\hline Baseline & $0.12 \pm 0.12$ & $0.13 \pm 0.10$ & 0.706 & 0.653 \\
\hline End & $0.59 \pm 0.30$ & $0.13 \pm 0.09$ & 0.000 & 0.000 \\
\hline$P 3$ & 0.000 & 0.998 & & \\
\hline \multicolumn{5}{|c|}{ Total grain (oz per $1000 \mathrm{kcal}$ ) } \\
\hline Baseline & $5.92 \pm 0.91$ & $6.87 \pm 0.89$ & 0.551 & 0.004 \\
\hline End & $7.30 \pm 0.72$ & $6.61 \pm 1.26$ & 0.000 & 0.000 \\
\hline$P 3$ & 0.049 & 0.063 & & \\
\hline \multicolumn{5}{|c|}{ Whole grain (oz per $1000 \mathrm{kcal}$ ) } \\
\hline Baseline & $0.32 \pm 0.07$ & $0.62 \pm 0.16$ & 0.000 & 0.000 \\
\hline End & $0.85 \pm 0.42$ & $0.28 \pm 1.04$ & 0.000 & 0.000 \\
\hline$P 3$ & 0.000 & 0.256 & & \\
\hline \multicolumn{5}{|c|}{ Milk (cup per $1000 \mathrm{kcal}$ ) } \\
\hline Baseline & $0.11 \pm 0.08$ & $0.10 \pm 0.04$ & 0.138 & 0.103 \\
\hline End & $0.61 \pm 0.244$ & $0.08 \pm 0.06$ & 0.000 & 0.000 \\
\hline$P 3$ & 0.000 & 0.242 & & \\
\hline \multicolumn{5}{|c|}{ Meet and beans (oz per $1000 \mathrm{kcal}$ ) } \\
\hline Baseline & $1.62 \pm 0.52$ & $1.66 \pm 0.73$ & 0.613 & 0.591 \\
\hline End & $1.76 \pm 0.56$ & $1.48 \pm 0.50$ & 0.030 & 0.025 \\
\hline$P 3$ & 0.318 & 0.130 & & \\
\hline \multicolumn{5}{|l|}{ Oils (gr per $1000 \mathrm{kcal}$ ) } \\
\hline Baseline & $9.81 \pm 4.74$ & $11.12 \pm 2.42$ & 0.553 & 0.127 \\
\hline End & $7.13 \pm 2.32$ & $13.80 \pm 3.75$ & 0.000 & 0.000 \\
\hline P3 & 0.009 & 0.000 & & \\
\hline \multicolumn{5}{|c|}{ Saturated fat (\% of energy) } \\
\hline Baseline & $12.85 \pm 2.13$ & $13.47 \pm 2.12$ & 0.268 & 0.419 \\
\hline End & $8.57 \pm 2.02$ & $13.31 \pm 1.80$ & 0.000 & 0.000 \\
\hline$P 3$ & 0.000 & 0.614 & & \\
\hline \multicolumn{5}{|c|}{ Sodium (gr per $1000 \mathrm{kcal}$ ) } \\
\hline Baseline & $1.82 \pm 1.20$ & $1.64 \pm 1.09$ & 0.554 & 0.356 \\
\hline End & $1.02 \pm 0.71$ & $1.72 \pm 0.97$ & 0.011 & 0.025 \\
\hline$P 3$ & 0.000 & 0.341 & & \\
\hline \multicolumn{5}{|l|}{ SoFAAS (\% of energy) } \\
\hline Baseline & $4.84 \pm 1.50$ & $6.72 \pm 2.32$ & 0.001 & 0.013 \\
\hline End & $5.17 \pm 2.07$ & $7.88 \pm 1.83$ & 0.000 & 0.000 \\
\hline$P 3$ & 0.432 & 0.044 & & \\
\hline
\end{tabular}

Data were expressed as mean \pm SD.

$P 1$ resulted from independent sample $t$-test; $P 2$ resulted from ANCONA test (adjustment for age and energy intake); and $P 3$ resulted from paired sample $t$ test. 
TABLE 5: Serum levels of inflammatory markers in the study groups at the baseline and end of study.

\begin{tabular}{lccr}
\hline Variables & Educated group $(n=30)$ & Noneducated group $(n=30)$ & $P 1$ \\
\hline Adiponectin & & & $P 2$ \\
Baseline & $8.52 \pm 2.28$ & $8.10 \pm 3.74$ & 0.609 \\
End & $11.72 \pm 4.24$ & $7.26 \pm 3.87$ & $\mathbf{0 . 0 1 3}$ \\
P3 & $\mathbf{0 . 0 2 7}$ & 0.330 & $\mathbf{0 . 0 3 5}$ \\
TNF- $\alpha$ & & & \\
Baseline & $10.05 \pm 4.60$ & $9.86 \pm 3.42$ & 0.809 \\
End & $7.91 \pm 2.46$ & $9.65 \pm 3.85$ & 0.858 \\
P3 & $\mathbf{0 . 0 3 1}$ & 0.605 & $\mathbf{0 . 0 4 4}$ \\
Hs-CRP & $7.50 \pm 3.01$ & & $\mathbf{0 . 0 1 1}$ \\
Baseline & $5.12 \pm 2.1$ & $7.00 \pm 3.43$ & 0.561 \\
End & $\mathbf{0 . 0 1 9}$ & $7.41 \pm 2.96$ & $\mathbf{0 . 0 2 1}$ \\
P3 & & 0.271 & $\mathbf{0 . 0 3 9}$ \\
\hline
\end{tabular}

Data were expressed as mean $\pm \mathrm{SD}$.

$P 1$ resulted from independent sample $t$-test; $P 2$ resulted from ANCONA test (adjustment for age, weight and energy intake); and $P 3$ resulted from paired sample $t$-test.

TABLE 6: Healthy eating index components and standards for scoring.

\begin{tabular}{lccl}
\hline Component & Maximum points & $\begin{array}{c}\text { Standard for maximum } \\
\text { score }\end{array}$ & Standard for minimum score of zero \\
\hline Total fruit (includes 100\% juice) & 5 & $\geq 0.8 \mathrm{cup} / 1000 \mathrm{kcal}$ & No fruit \\
Whole fruit (not juice) & 5 & $\geq 0.4 \mathrm{cup} / 1000 \mathrm{kcal}$ & No whole fruit \\
Total vegetables & 5 & $\geq 1.1 \mathrm{cups} / 1000 \mathrm{kcal}$ & No vegetables \\
Dark green and orange & 5 & $\geq 0.4 \mathrm{cup} / 1000 \mathrm{kcal}$ & No dark green or orange vegetables or \\
vegetables and legumes & 5 & $\geq 3.0 \mathrm{cups} / 1000 \mathrm{kcal}$ & No grains \\
Total grains & 5 & $\geq 1.5 \mathrm{oz} / 1000 \mathrm{kcal}$ & No whole grains \\
Whole grains & 10 & $\geq 1.3 \mathrm{cups} / 1000 \mathrm{kcal}$ & No milk \\
Milk & 10 & $\geq 2.5 \mathrm{oz} / 1000 \mathrm{kcal}$ & No meat or beans \\
Meat and beans & 10 & $\geq 12 \mathrm{grams} / 1000 \mathrm{kcal}$ & No oil \\
Oils & 10 & $\leq 7 \%$ of energy & $\geq 15 \%$ of energy \\
Saturated fat & 10 & $\leq 0.7 \mathrm{gram} / 1000 \mathrm{kcal}$ & $\geq 2.0$ grams $/ 1000 \mathrm{kcal}$ \\
Sodium & 20 & $\leq 20 \%$ of energy & $\geq 50 \%$ of energy \\
Calories from solid fat, alcohol, & & & \\
and added sugar (SoFAAS) & & &
\end{tabular}

folate, vitamin C, beta carotene, selenium, magnesium, and cupper, which was found mainly in the HEI components (fruits, vegetables, legumes, and whole grains), are positively associated with improved metabolic responses and have beneficial effects on markers of inflammation [25, 26]. However, since foods are not eaten in isolation, recent studies affirmed the importance of the overall dietary pattern or dietary quality, rather than that of specific food groups or nutrients, to inflammatory markers [22]. Although, in the present study, the relation between total HEI components scores and inflammatory markers was not assessed, individuals with higher total HEI scores had significantly higher adiponectin concentration and lower hs-CRP and TNF- $\alpha$ level (Table 6). In this study, it was attempted to account for confounders that were likely associated with concentration of inflammatory markers or consumption of a diet. However, the potential for remaining confounders by uncontrolled covariates was possible, and the present study was also limited by the small sample size, short duration of the intervention, and the self-report of food intake. Finally, the sample used in this randomized clinical trial does not represent a random sample of the Iran population; thus future studies with larger sample size are needed to identify these determinants.

\section{Implications for Research and Practice}

The results of this study support the hypothesis that beneficial effects of improved dietary quality with respect to obesity may be partially mediated by improvements in plasma concentrations of adiponectin and other biomarkers of systemic inflammation. This effect could be independent of weight loss and the other anthropometric variations. Future studies should extend these findings by investigating potential mechanisms underlying these relations and by examining whether prevention of the diabetes, atherosclerosis, and metabolic syndrome by diet quality improvements is mediated through changes 
in clinically important biomarkers, including adiponectin, hs-CRP, and TNF- $\alpha$ concentration.

\section{Conflict of Interests}

The authors declare that there is no conflict of interests regarding the publication of this paper.

\section{Acknowledgments}

This study is issued from the Master of Science thesis for Sara Ebrahimi. Special thanks are due to Arvand International Division of Ahvaz Jundishapur University of Medical Sciences for the financial support (Grant no. B-9115).

\section{References}

[1] A. Boynton, M. L. Neuhouser, B. Sorensen, A. McTiernan, and C. M. Ulrich, "Predictors of diet quality among overweight and obese postmenopausal women," Journal of the American Dietetic Association, vol. 108, no. 1, pp. 125-130, 2008.

[2] J. L. Fargnoli, T. T. Fung, D. M. Olenczuk, J. P. Chamberland, F. B. Hu, and C. S. Mantzoros, "Adherence to healthy eating patterns is associated with higher circulating total and highmolecular-weight adiponectin and lower resistin concentrations in women from the Nurses' Health Study," The American Journal of Clinical Nutrition, vol. 88, no. 5, pp. 1213-1224, 2008.

[3] A. Drewnowski, E. C. Fiddler, L. Dauchet, P. Galan, and S. Hercberg, "Diet quality measures and cardiovascular risk factors in France: applying the healthy eating index to the SU.VI.MAX study," Journal of the American College of Nutrition, vol. 28, no. 1, pp. 22-29, 2009.

[4] S. M. George, M. L. Neuhouser, S. T. Mayne et al., "Postdiagnosis diet quality is inversely related to a biomarker of inflammation among breast cancer survivors," Cancer Epidemiology Biomarkers and Prevention, vol. 19, no. 9, pp. 2220-2228, 2010.

[5] S. Y. Bu, "Transitional changes in energy intake, skeletal muscle content and nutritional behavior in college students during course-work based nutrition education," Clinical Nutrition Research, vol. 2, no. 2, pp. 125-134, 2013.

[6] A. P. Tardivo, J. Nahas-Neto, E. A. P. Nahas, N. Maesta, M. A. H. Rodrigues, and F. L. Orsatti, "Associations between healthy eating patterns and indicators of metabolic risk in postmenopausal women," Nutrition Journal, vol. 9, no. 1, article 64, 2010.

[7] N. A. Tek, H. Yildiran, G. Akbulut et al., "Evaluation of dietary quality of adolescents using Healthy Eating Index," Nutrition Research and Practice, vol. 5, no. 4, pp. 322-328, 2011.

[8] A. Boynton, M. L. Neuhouser, M. H. Wener et al., "Associations between healthy eating patterns and immune function or inflammation in overweight or obese postmenopausal women," American Journal of Clinical Nutrition, vol. 86, no. 5, pp. 14451455, 2007.

[9] A. K. Kant and B. I. Graubard, "A comparison of three dietary pattern indexes for predicting biomarkers of diet and disease," Journal of the American College of Nutrition, vol. 24, no. 4, pp. 294-303, 2005.

[10] M. L. Neuhouser, R. E. Patterson, I. B. King, N. K. Horner, and J. W. Lampe, "Selected nutritional biomarkers predict diet quality," Public Health Nutrition, vol. 6, no. 7, pp. 703-709, 2003.
[11] E. Kennedy, "Putting the pyramid into action: the healthy eating index and food quality score," Asia Pacific Journal of Clinical Nutrition, vol. 17, supplement 1, pp. 70-74, 2008.

[12] S. Taechangam, U. Pinitchun, and C. Pachotikarn, "Development of nutrition education tool: healthy eating index in Thailand," Asia Pacific Journal of Clinical Nutrition, vol. 17, supplement 1, pp. 365-367, 2008.

[13] E. S. Ford, A. H. Mokdad, and S. Liu, "Healthy Eating Index and C- reactive protein concentration: findings from the National Health and Nutrition Examination Survey III, 1988-1994," European Journal of Clinical Nutrition, vol. 59, no. 2, pp. 278-283, 2005.

[14] T. T. Fung, M. L. McCullough, P. K. Newby et al., "Diet-quality scores and plasma concentrations of markers of inflammation and endothelial dysfunction," The American Journal of Clinical Nutrition, vol. 82, no. 1, pp. 163-173, 2005.

[15] L. Azadbakht, P. Mirmiran, F. Hosseini, and F. Azizi, "Diet quality status of most Tehranian adults needs improvement," Asia Pacific Journal of Clinical Nutrition, vol. 14, no. 2, pp. 163168, 2005.

[16] S. J. Woodruff, R. M. Hanning, K. McGoldrick, and K. S. Brown, "Healthy eating index-C is positively associated with family dinner frequency among students in grades 6-8 from Southern Ontario, Canada," European Journal of Clinical Nutrition, vol. 64, no. 5, pp. 454-460, 2010.

[17] K. M. Hurley, S. E. Oberlander, B. C. Merry, M. M. Wrobleski, A. C. Klassen, and M. M. Black, "The healthy eating index and youth healthy eating index are unique, nonredundant measures of diet quality among low-income, African American adolescents," Journal of Nutrition, vol. 139, no. 2, pp. 359-364, 2009.

[18] S. C. de Andrade, M. B. de Azevedo Barros, L. Carandina, M. Goldbaum, C. L. G. Cesar, and R. M. Fisberg, "Dietary quality index and associated factors among adolescents of the state of Sao Paulo, Brazil," Journal of Pediatrics, vol. 156, no. 3, pp. 456460, 2010.

[19] P. Togo, M. Osler, T. I. A. Sørensen, and B. L. Heitmann, "Food intake patterns and body mass index in observational studies," International Journal of Obesity, vol. 25, no. 12, pp. 1741-1751, 2001.

[20] A. Esmaillzadeh and L. Azadbakht, "Dairy consumption and circulating levels of inflammatory markers among Iranian women," Public Health Nutrition, vol. 13, no. 9, pp. 1395-1402, 2010.

[21] E. Lopez-Garcia, M. B. Schulze, T. T. Fung et al., "Major dietary patterns are related to plasma concentrations of markers of inflammation and endothelial dysfunction," The American Journal of Clinical Nutrition, vol. 80, no. 4, pp. 1029-1035, 2004.

[22] C. S. Mantzoros, C. J. Williams, J. E. Manson, J. B. Meigs, and F. B. Hu, "Adherence to the Mediterranean dietary pattern is positively associated with plasma adiponectin concentrations in diabetic women," The American Journal of Clinical Nutrition, vol. 84, no. 2, pp. 328-335, 2006.

[23] C. Chrysohoou, D. B. Panagiotakos, C. Pitsavos, U. N. Das, and C. Stefanadis, "Adherence to the Mediterranean diet attenuates inflammation and coagulation process in healthy adults: the ATTICA study," Journal of the American College of Cardiology, vol. 44, no. 1, pp. 152-158, 2004.

[24] K. Esposito, A. Pontillo, C. Di Palo et al., "Effect of weight loss and lifestyle changes on vascular inflammatory markers in obese women: a randomized trial," The Journal of the American Medical Association, vol. 289, no. 14, pp. 1799-1804, 2003. 
[25] N. M. McKeown, J. B. Meigs, S. Liu, E. Saltzman, P. W. F. Wilson, and P. F. Jacques, "Carbohydrate nutrition, insulin resistance, and the prevalence of the metabolic syndrome in the framingham offspring cohort," Diabetes Care, vol. 27, no. 2, pp. 538-546, 2004.

[26] N. M. McKeown, J. B. Meigs, S. Liu, P. W. F. Wilson, and P. F. Jacques, "Whole-grain intake is favorably associated with metabolic risk factors for type 2 diabetes and cardiovascular disease in the Framingham Offspring Study," The American Journal of Clinical Nutrition, vol. 76, no. 2, pp. 390-398, 2002. 


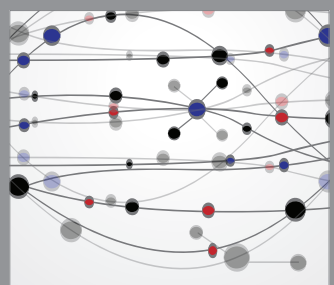

The Scientific World Journal
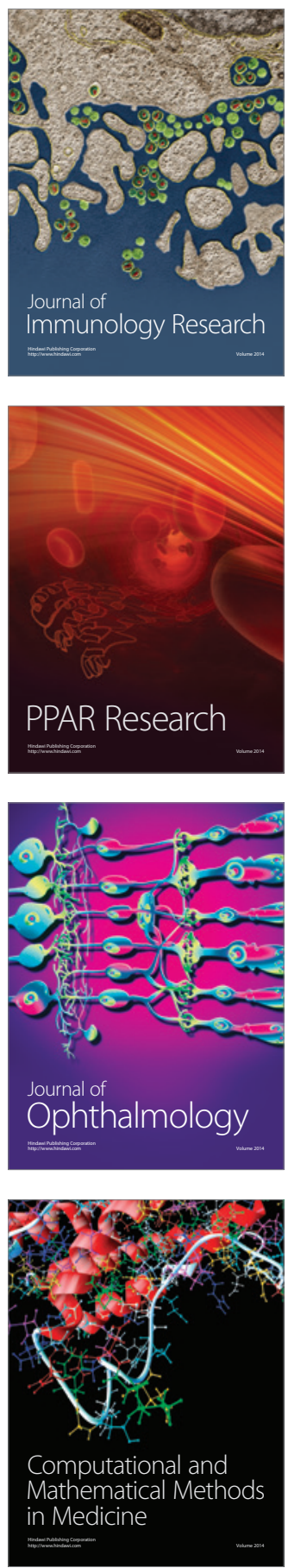

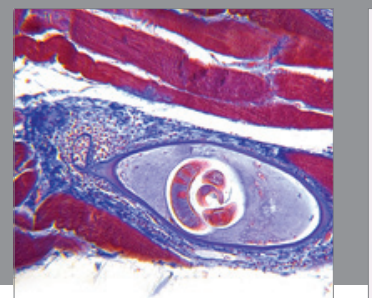

Gastroenterology

Research and Practice
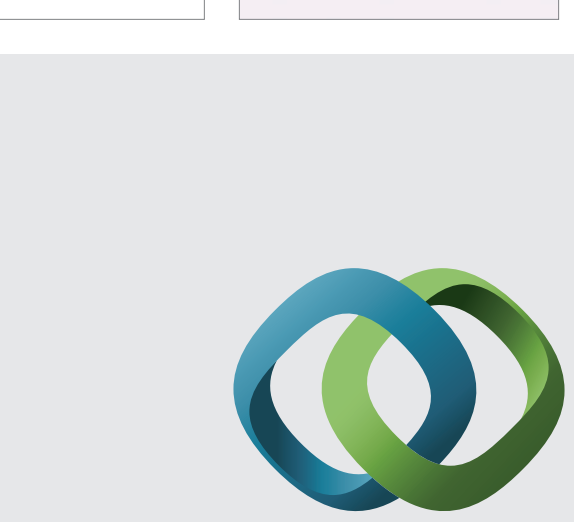

\section{Hindawi}

Submit your manuscripts at

http://www.hindawi.com
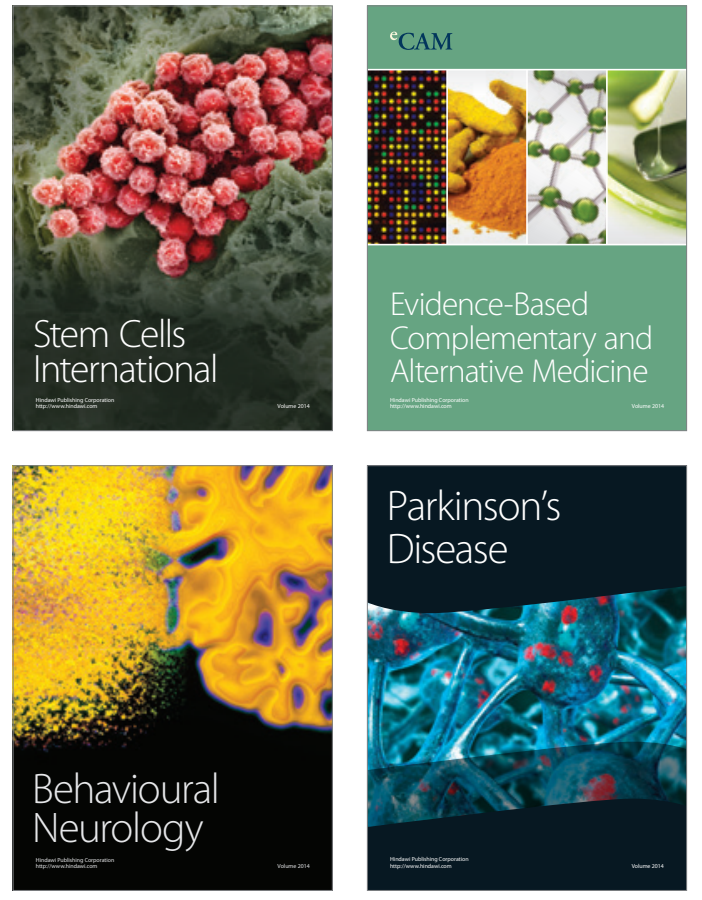
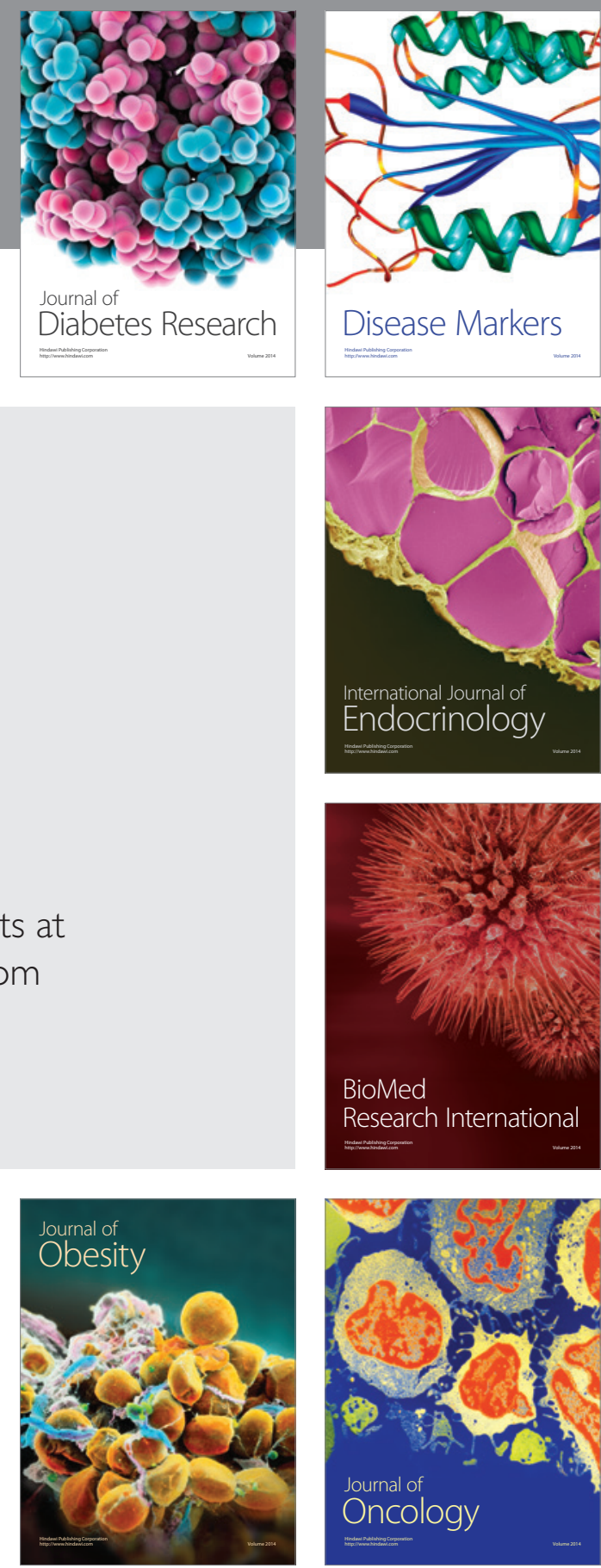

Disease Markers
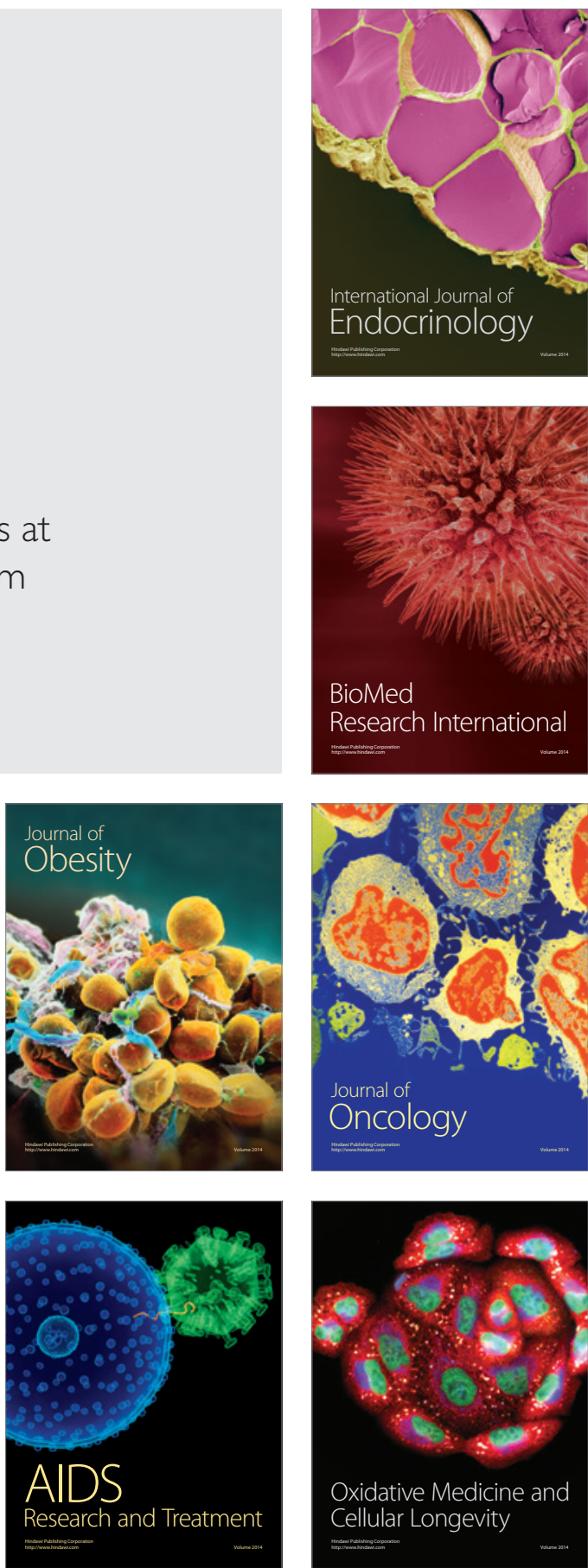